\title{
Insulin-loaded polymeric mucoadhesive nanoparticles: development, characterization and cytotoxicity evaluation
}

\author{
Tiago Henrique Honorato Gatti ${ }^{1 \#}$, Josimar Oliveira Eloy ${ }^{1 \#^{* *}}$, Leonardo Miziara Barboza Ferreira ${ }^{1}$, \\ Isabel Cristine da Silva², Fernando Rogério Pavan², Maria Palmira Daflon Gremião ${ }^{1}$, Marlus Chorilli1* \\ ${ }^{1}$ School of Pharmaceutical Sciences, UNESP - São Paulo State University, Campus Araraquara, Department of Drugs and \\ Medicines, Araraquara, SP, Brazil, ${ }^{2}$ School of Pharmaceutical Sciences, UNESP - São Paulo State University, Campus \\ Araraquara, Department of Biological Sciences, Araraquara, SP, Brazil
}

\begin{abstract}
Mucoadhesive nanoparticles are particularly interesting for delivery through nasal or pulmonary routes, as an approach to overcome the mucociliary clearance. Moreover, these nanoparticles are attractive for peptide and protein delivery, particularly for insulin to treat diabetes, as an alternative to conventional parenteral administration. Thus, chitosan, a cationic mucoadhesive polysaccharide found in shells of crustaceans, and the negatively-charged dextran sulfate are able to form nanoparticles through ionic condensation, representing a potential insulin carrier. Herein, chitosan/dextran sulfate nanoparticles at various ratios were prepared for insulin loading. Formulations were characterized for particle size, zeta potential, encapsulation efficiency, scanning electron microscopy, differential scanning calorimetry, and in vitro drug release. Moreover, the interaction with mucin and the cytotoxicity against a lung cell line were studied, which altogether have not been addressed before. Results evidenced that a proper selection of polyelectrolytes is necessary for smaller particle size formation and also the composition and zeta potential impact encapsulation efficiency, which is benefited by the positive charge of chitosan. Insulin remained stable after encapsulation as evidenced by calorimetric assays, and was released in a sustained manner in the first $10 \mathrm{~h}$. Positively-charged nanoparticles based on chitosan/dextran-sulfate at the ratio of 6:4 successfully interacted with mucin, which is a prerequisite for delivery to mucus-containing tissues. Finally, insulin-loaded nanoparticles displayed no cytotoxicity effect against lung cells at tested concentrations, suggesting the potential for further in vivo studies.
\end{abstract}

Keywords: Insulin. Chitosan. Dextran-Sulfate. Nanoparticles. Mucoadhesion.

\section{INTRODUCTION}

Diabetes mellitus represents the most prevalent metabolic disorder nowadays, with 345 million people affected worldwide (Sah et al., 2016). Furthermore, it is believed that in 2030 the number of patients will raise up to 552 million, which can be considered a threat for public health (Whiting et al., 2011). A major concern is that life expectancy is reduced by many years in patients with type 1 or 2 diabetes. The therapy involves different approaches, including diet, physical exercise and hypoglycemic drugs.

\footnotetext{
*Correspondence: M. Chorilli. Faculdade de Ciências Farmacêuticas, Departamento de Fármacos e Medicamentos, Universidade Estadual Paulista (UNESP). Rodovia Araraquara-Jau, km. 1, 14801-902, Araraquara, São Paulo, Brasil. Telefone.: +55 163301 6998. E-mail: chorilli@fcfar.unesp.br / J. O. Eloy: josimar.eloy@gmail.com

" Both authors equally contributed to this paper
}

For Type 1 diabetes patients, due to insufficient insulin production, exogenous hormone is needed (Salvioni et al., 2016). The peptide insulin is the most effective drug for diabetes treatment, with high specificity and activity (Fonte et al., 2014). However, the most common route for insulin administration, the parenteral route, faces many hurdles, such as the difficulty of achieving a normal pattern of nutrient-related and basal insulin. Furthermore, the subcutaneous injection, which must pass through the skin for systemic effect, results in considerable tissue trauma and pain (Sintov, Levy, Botner 2010; Li et al., 2017).

Considering the instability of peptides, for an effective therapeutic outcome, they should be protected against degradation. Therefore, the use of appropriate carriers is needed for effective delivery (Huang et al., 2009). Strategies have been directed to improve insulin delivery by the use of colloidal systems, possessing diameter less than $1 \mu \mathrm{m}$ (Diop 
et al., 2015). Among these colloidal carriers, nanoparticles are able to prevent peptide degradation and promote sustained release, with consequent better therapeutic response and patient compliance (Zheng et al., 2013; Giovino et al., 2012). Thus, nanoparticles have been used for nasal or pulmonary routes for delivery of insulin, avoiding the problems associated with the parenteral administration. For instance, the nasal route of delivery has been exploited for needle-free systemic delivery of a wide range of drugs, including small molecules, proteins and peptides, such as insulin. Noteworthy, intranasal drug delivery offers many advantages, such as the large absorptive area with high vascularization, avoiding the first-pass liver metabolism (Casettari, Illum 2014; Zhang et al., 2008). Furthermore, the pulmonary route is also interesting for drug delivery, such as insulin, due to the large surface area for drug absorption, which can also benefit from mucoadhesive formulations, especially those capable of overcoming the mucociliary clearance (Jain et al., 2008; Alpar et al., 2005).

Bioadhesive polymers, for example chitosan and alginate, are polysaccharides employed in nanoparticle formation owing to their mucoadhesive properties, through the interaction with the negatively charged mucin (Andreani et al., 2015). Chitosan, a cationic polysaccharide with glucosamine and $\mathrm{N}$-acetylglucosamine, is derived from the deacetylation of chitin, found in shells of crustaceans and is soluble in mildly acidic aqueous solutions. Chitosan presents many advantages, such as its availability and biocompatibility. Owing to its many advantages, chitosan has been used as a drug delivery carrier, including for controlled release of insulin. Very interestingly, chitosan hydration and gel formation allow to prolong release of the drug at the administration site (Huang et al., 2009; Ravindranathan et al., 2016; Szekalska et al., 2016). Moreover, chitosan is able to disrupt epithelium tight junctions, due to interaction of protein Kinase C (Smith, Dornish, Wood, 2005). Although polyelectrolyte nanoparticles based on chitosan have been previously reported for insulin loading, to our knowledge, our paper is the first to address the in vitro mucoadhesion or cytotoxicity on lung fibroblast cells of chitosan/sulfate dextran nanoparticles loaded with insulin. Altogether, these are important preliminary parameters regarding pulmonary or nasal delivery of insulin from nanoparticles (Sarmento, Veiga, Ferreira, 2006; Mao et al., 2006; Lopes et al., 2016). Recently, the effect of albumin coating on chitosan/dextran-sulfate nanoparticles was investigated. The authors aimed to protect insulin from degradation in the acidic or intestinal environments considering the oral drug delivery. Unlike our study, the authors employed the nanoemulsion method for nanoparticle preparation, using surfactants for stabilization, such as sorbitane monooleate and Poloxamer 188 (Lopes et al., 2016).

Thus, the purpose of this work was to develop and characterize formulations based on chitosan/ dextran-sulfate, intended for mucoadhesion of insulin, potentially applied for nasal or pulmonary delivery. We investigated the chitosan/dextran-sulfate ratio influence on encapsulation efficiency, zeta potential and particularly on particle size. Additionally, we studied the in vitro drug release using dialysis membrane and the interaction between the formulation and insulin through thermal analysis employing the NanoDSC equipment. Noteworthy, the complexation with mucin for in vitro mucoadhesive evaluation and the cytotoxicity of formulations against MCR-5 fibroblast lung cells, both important parameters prior to in vivo application, were addressed.

\section{MATERIAL AND METHODS}

\section{Material}

Dextran sulfate sodium salt (average MW $>500,000$ $\mathrm{Da}$ ), chitosan (low molecular weight. MW, ranging from 50,000 to190,000 Da), resazurin, porcine stomach mucin, type II, and doxorubicin were purchased from Sigma-Aldrich. Novolin ${ }^{\circledR} \mathrm{R}$ human insulin was obtained from Novo Nordisk. Glacial acetic acid was supplied by QUEMIS. 12-14 kDa MWCO (molecular weight cutoff) cellulose dialysis membranes were obtained from Fisherbrand. MRC-5 cells were supplied by American Type Collection. DMEM medium, fetal bovine serum (FBS) and antibiotic/antimicotic solution were purchased from Gibco. 0.25\% trypsin/EDTA was supplied by Vitrocell. Ultra-purified water was obtained from Milli ${ }^{\circledR} \mathrm{Q}$ Plus System (Millipore).

\section{Methods}

\section{Development of insulin-loaded nanoparticles}

The nanoparticles were synthesized by the combination of two polymers, a polycation, chitosan (low molecular weight. MW, ranging from 50,000 to $190,000 \mathrm{Da})$, and a polyanion, dextran-sulfate sulfate sodium salt (average MW $>500,000 \mathrm{Da}$ ) (Sarmento, Veiga, Ferreira, 2006). The total polymer concentration, 0.5, 1.0 , and $1.5 \mathrm{mg} / \mathrm{mL}$ in aqueous solution, $\mathrm{pH} 4,6$, and the chitosan/dextran-sulfate ratio, $1: 9,2: 8,3: 7,4: 6,1: 1,6: 4$, 7:3, 8:2 and 9:1, were investigated. Nanoparticles were obtained after dropwise addition of chitosan solution to dextran-sulfate solution under vortex stirring for $15 \mathrm{~min}$. Insulin was loaded in the formulations at $500 \mu \mathrm{g} / \mathrm{mL}$ 
concentration. For this purpose, the peptide addition order was studied, i.e, after the formation of the nanoparticle, or onto chitosan solution, or onto dextran sulfate solution, prior to the nanoparticle formation.

\section{Physicochemical characterization of insulin-loaded nanoparticles}

\section{- Particle size}

Particle size was measured through the dynamic light scattering (DLS) technique at $633 \mathrm{~nm}$, using the angle of $173^{\circ}$, at room temperature (Zetasizer Nano-Zs, Malvern Instruments). Samples were diluted with water before analysis. Measurements were done in triplicate with 10 determinations for each one.

\section{- Zeta potential}

Zeta potential was measured through electrophoretic mobility of particles. Analysis was carried out using the DLS equipment (Zetasizer Nano-Zs, Malvern Instruments). Samples were diluted with water before analysis. Measurements were done in triplicate with 10 determinations for each one.

- Encapsulation efficiency

The encapsulation efficiency (EE) was determined by ultraviolet-visible (UV) spectroscopy, with insulin measurement at $270 \mathrm{~nm}$, following equation 1 . For separation of insulin-loaded nanoparticle and free insulin, centrifugation was performed at $14.000 \mathrm{rpm}$ for $10 \mathrm{~min}$. Free insulin corresponded to the insulin in the supernatant fraction (Sarmento, Veiga, Ferreira 2006).

$(\mathrm{EE} \%)=\frac{\text { Total insulin }(\mu \mathrm{g})-\text { Free insulin }(\mu \mathrm{g})}{\text { Total insulin }(\mu \mathrm{g})}$ (Equation 1)

- Differential Scanning Calorimetry (DSC)

DSC analysis was carried out using the Nano DSC equipment, TA Instruments. Samples were degassed under vacuum before loading into the capillary cells composed by platinum and heated from 0 to $100^{\circ} \mathrm{C}$, at a rate of $2^{\circ} \mathrm{C} / \mathrm{min}$. The samples scans were subtracted from ultra-purified water reference scan. Data analyses were carried out using the Nanoanalyse software (Andreani et al., 2015).

- Morphology by Scanning Electron Microscopy (SEM)

SEM morphology was evaluated using a JEOL JSM-7500F microscope. For that, a drop of nanoparticle dispersion was applied to the carbon grid, dried and gold coated under vacuum. Photomicrographs were obtained using $2.00 \mathrm{kV}$ electron beam.

\section{In vitro insulin release}

Experiments were done in sextuplicate at $32{ }^{\circ} \mathrm{C}$ using a Franz diffusion cell system, at $400 \mathrm{rpm}$. Cellulose acetate membranes (12-14 kDa MWCO) were placed between the donor and the receptor compartments, the latter filled with deionized water $(7 \mathrm{~mL})$, following a method previously described, with modifications (Liu et al., 2012). Samples in the receptor compartments were collected in predetermined intervals, with replacement of fresh medium. Released insulin was quantified in the receptor compartment, using a spectrophotometric method at $270 \mathrm{~nm}$, using an insulin standard curve.

\section{In vitro mucin/ nanoparticle interaction}

Blank nanoparticles were incubated with mucin solution $(2 \mathrm{mg} / \mathrm{mL})$ at different mucin/nanoparticles ratios, vortexed and incubated under stirring at $37^{\circ} \mathrm{C}$ for $30 \mathrm{~min}$ (Andreani et al., 2015). Afterwards, the dispersions were centrifuged for $10 \mathrm{~min}$ at $10000 \mathrm{rpm}$ and the supernatant was used for mucin UV spectrophotometric quantification at $280 \mathrm{~nm}$ (Boya et al., 2017). Mucin adsorbed on samples was determined by the difference between the final and initial mucin concentration after incubation and centrifugation.

\section{Cell culture}

MRC-5 (normal lung fibroblast cells) cell line was obtained from the American Type Culture Collection (Manassas, VA, USA) and incubated in DMEM medium supplemented with $10 \% \mathrm{FBS}$ and $1 \%$ penicillin $(100 \mathrm{U} /$ $\mathrm{mL})$-streptomycin $(100 \mu \mathrm{g} / \mathrm{mL})$. Cells were maintained in a humidified environment at $37^{\circ} \mathrm{C}$ with $5 \% \mathrm{CO}_{2}$ and sub-cultured twice per week.

\section{Cytotoxicity tests}

A resazurin reduction assay was used to investigate cytotoxicity on MRC-5 cells. The assay is based on reduction of the indicator dye, resazurin, to the highly fluorescent resorufin by viable cells. Nonviable cells rapidly lose the metabolic capacity to reduce resazurin and thus do not produce a fluorescent signal. Briefly, the cells were detached by treatment with $0.25 \%$ trypsin/ EDTA (VitroCell, Brazil) and $2.5 \times 10^{4}$ cells were placed on each well of a 96-well cell culture plate (Costar, USA) in a total volume of $100 \mu \mathrm{L}$. Cells were allowed to adhere overnight and then were treated with different 
concentrations of drugs. After $24 \mathrm{~h}$ incubation in the presence of the compounds, the medium was removed and $50 \mu \mathrm{L}$ resazurin (Sigma-Aldrich, Germany) $0.01 \% \mathrm{w} / \mathrm{v}$ in DMEM, was added to each well and the plates were incubated at $37^{\circ} \mathrm{C}$ for $3 \mathrm{~h}$.

The fluorescence was measured on Biotek Synergy H1 plate reader (Biotek, Winooski, VT) using an excitation wavelength of $530 \mathrm{~nm}$ and an emission wavelength of 590 $\mathrm{nm}$. Untreated cells constituted the negative control (viable cells), and cells treated with doxorubicin at $100 \mathrm{nmol}$ (Sigma-Aldrich, St. Louis, MO, USA) constituted the positive control (dead cells). All the tests were performed in three independent assays. Graphs were expressed as lethality (\%). The $\mathrm{IC}_{50}$ values represent the samples concentrations required to inhibit $50 \%$ of cell proliferation and were calculated using the GraphPad Prism ${ }^{\circledR} 5$ (Version 5.01, GraphPad Software, Inc., USA)

\section{RESULTS AND DISCUSSION}

Polyelectrolyte complexes can be synthesized in aqueous solutions through ionic interactions between polyelectrolyte compounds with opposite charges, particularly in low ionic strength media. Critical coagulation concentrations of 0.12 and $0.09 \mathrm{M}$ (with sodium chloride) for cationic and anionic particles has evidenced a mostly electrostatic stabilization (Schatz et al., 2004a). Furthermore, dissociation of complexes decreases with increased salt concentration, which is likely due to the result of reduced attraction between the oppositely charged polyelectrolytes (Mao et al., 2006). For complexation with chitosan, dextran sulfate is the most widely employed polysaccharide, due to many advantages, such as the low price, ease availability and also because of the strong ionic interaction between its sulfate group and the ammonium groups of chitosan (Delair, 2011). Nanoparticles have been shown to be formed through the coacervation between chitosan and dextran sulfate, with application for the loading of proteins and peptides, such as insulin (Sarmento, Veiga, Ferreira, 2006). Furthermore, the ionic coacervation is environment friendly and the results obtained over the years with nanoparticles based on chitosan and dextran sulfate underline the high potential of this strategy for development of human medicine (Delair, 2011).

Figure 1 summarized the results of particle size obtained with different nanoparticle compositions, containing decreasing total excipient concentrations, prepared at varied chitosan/dextran sulfate ratios. It became evident that unless prepared with only chitosan or dextran sulfate, nanoparticles composed by the mixture of the two polyelectrolytes were not strongly affected by the ratio between the components. Conversely, Shatz and co-workers(2004b) observed that higher dextran sulfate concentrations led to increased particle size, whereas higher concentration of chitosan favored the formation of more particles, without influencing particle size. However, the nanoparticles were prepared differently compared to the protocol employed herein, which could potentially influence the particle size results obtained. It was reported the use of a purification step of nanoparticles by centrifugation for $30 \mathrm{~min}$ at $8000-12000 \mathrm{rpm}$, with resuspension in a minimum volume of deionized water, in order to avoid the adsorption of free polymer onto particle surface. Also, we need to consider that the insulin presence in the nanoparticles reported in our paper could have changed the behavior observed in the paper by Schatz et al.(2004b), who did not encapsulate the peptide.
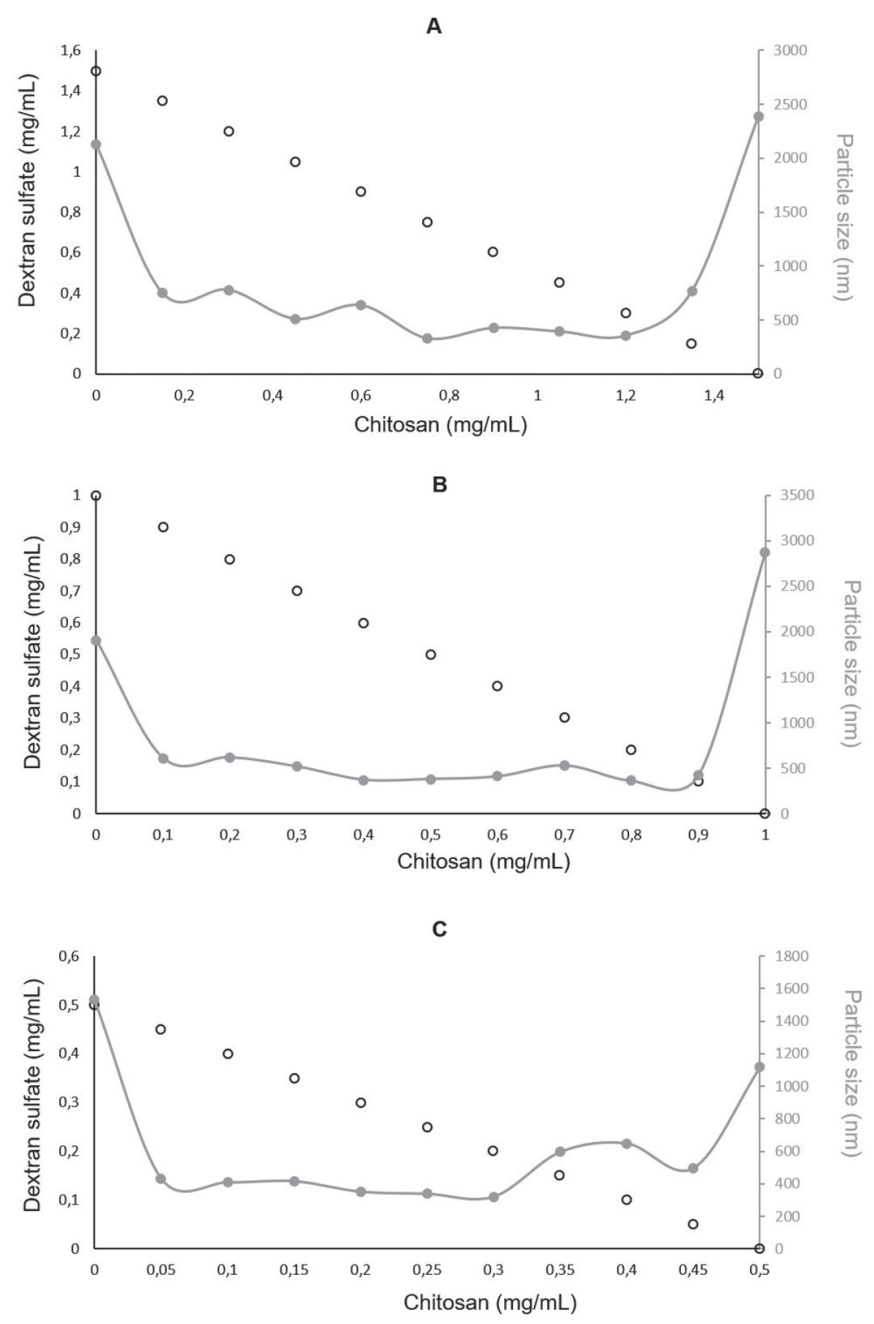

FIGURE 1 - Study of the influence of total excipient concentration $(\mathrm{A}=1.5 \mathrm{mg} / \mathrm{mL}, \mathrm{B}=1.0 \mathrm{mg} / \mathrm{mL}$ and $\mathrm{C}=0.5 \mathrm{mg} / \mathrm{mL})$ and the ratio of dextran sulfate/chitosan (open circle) on particle size (gray closed circle). 
Noteworthy, herein we found that the total polymer concentration affected the particle size. Chitosan and dextran sulfate concentrations equivalent to $0.5,1.0$ and $1.5 \mathrm{mg} / \mathrm{mL}$ resulted in mean particle size values of all formulations at $605.06,816.30$ and $859 \mathrm{~nm}$, respectively. Therefore, the total polymer concentration chosen for further studies was $0.5 \mathrm{mg} / \mathrm{mL}$ and the selected formulations were prepared employing chiton/dextran sulfate at the ratios of 1:9, with particle size of 432.2 $\mathrm{nm}$ and zeta potential of $-54.7 \pm 0.458 \mathrm{mV}$, due to the negatively charged dextran sulfate in excess, and another composition with chitosan in excess, at the ratio of $6: 4$, generating particle size of $320.55 \mathrm{~nm}$ and zeta potential of $38.6 \pm 0.1 \mathrm{mV}$. Interestingly, particle size achieved with this latter formulation developed herein is smaller than the chitosan/dextran sulfate particles previously reported for insulin loading, which ranged from $489 \pm 11$ to 1612 \pm 248 (Sarmento, Veiga, Ferreira, 2006). The reduction of particle size is important for further applications of the nanoparticles developed herein. For instance, several types of nanoparticles have been employed for lung drug delivery with improved uptake and action and it is known that smaller nanoparticles traverse the surfactant layer more efficiently than larger nanoparticles probably due to minimal steric hindrance (Iyer, Hsia, Nguyen, 2015).

The encapsulation efficiency of insulin in nanoparticles was low, equivalent to 9.73 and $16.67 \%$, for the chitosan:dextran sulfate (1:9) and chitosan:dextran sulfate $(6: 4)$ formulations, respectively, in disagreement with previous observations of high encapsulation efficiency of insulin in formulations prepared with excess dextran sulfate (Sarmento, Veiga, Ferreira, 2006). In order to circumvent this drawback, it was attempted to add insulin in the chitosan solution or in the dextran sulfate solution, prior to the nanoparticle coacervation, albeit even lower encapsulation efficiency values were achieved (data not shown). Within this context, Mao and co-workers proved that the complexation between chitosan and insulin is $\mathrm{pH}$ dependent, and it is favored above the critical $\mathrm{pH}$ of 6.0 (Mao et al., 2006). On the other hand, in that paper, the formulation was different than the one reported herein, because it was not used dextran sulfate, a counterion commonly employed to form nanoparticles though ionic complexation (Chaiyasan, Srinivas, Tiyaboonchai 2013). Furthermore, the $\mathrm{pH}$ requirement for nanoparticle formation when dextran-sulfate is used in combination with chitosan is different, considering that Sarmento, Veiga and Ferreira (2006) prepared insulin-loaded nanoparticles in $\mathrm{pH}$ below 5.0 with excellent encapsulation efficiency, higher than $85 \%$. However, we need to consider that while Sarmento, Veiga and Ferreira (2006) used insulin at a concentration of $58.33 \mu \mathrm{g} / \mathrm{mL}$, whereas we employed a higher drug concentration of $500 \mu \mathrm{g} / \mathrm{mL}$, which could have affected the encapsulation efficiency due to insulin excess. Hence, the insulin concentration of our nanoparticles was almost 10 times higher, which could compensate the low encapsulation efficiency of insulin. Finally, the low encapsulation of hydrophilic drugs in polymeric nanoparticles has been commonly reported and remains a challenge during formulation development (Vrignaud, Benoit, Saulnier, 2011). Therefore, the low encapsulation efficiency shortcoming should be addressed in more details in future studies.

The evaluation of the morphology properties of the formulations was conducted by SEM. Photomicrographs shown in Figure 2 evidenced the aggregate nature of spherical chitosan/dextran sulfate (1:9) nanoparticles, unlike with the chitosan/dextran sulfate (6:4) formulation, which did not present defined structure, probably to excess adhesive chitosan which resulted and highly aggregated formulation.

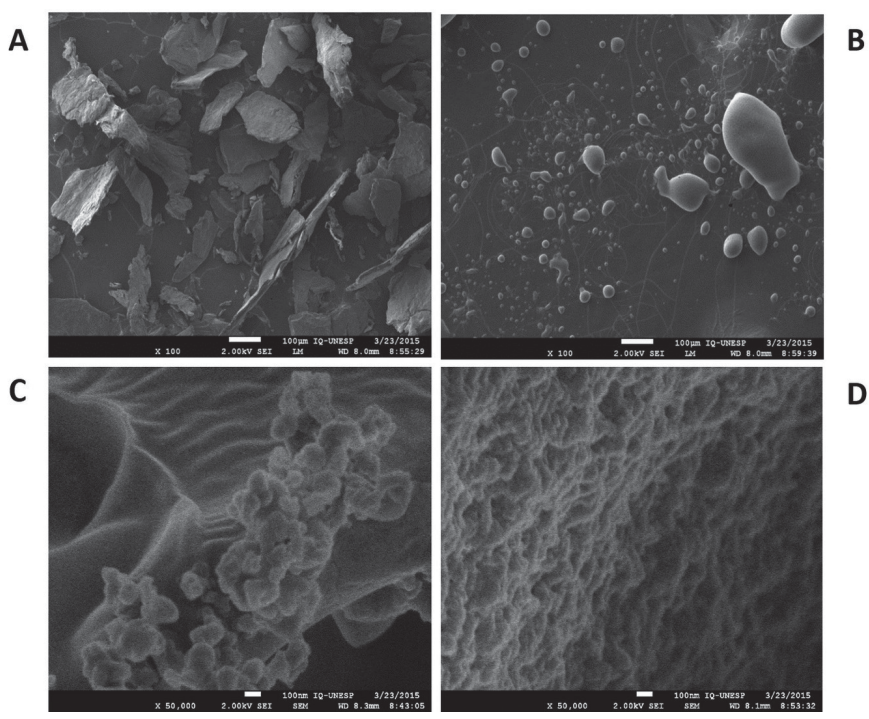

FIGURE 2 - Scanning electron microscopy (SEM) of A (dextran sulfate), B (chitosan), C (chitosan/dextran sulfate (1:9) nanoparticles) and D (chitosan/dextran sulfate (6:4) nanoparticles).

DSC experiments can reveal structural changes of proteins when incorporated into delivery systems and was therefore carried out to elucidate the thermal events of insulin-loaded nanoparticles (Jorgensen et al., 2004). All the endothermic and exothermic insulin peaks were indicated with arrows. Figure 03 revealed a minor endothermic peak centered at $77^{\circ} \mathrm{C}\left(\Delta \mathrm{H}=0.04 \mathrm{~kJ} \mathrm{~mol}^{-1}\right)$ followed by a major exothermic peak at $85.30^{\circ} \mathrm{C}(\Delta \mathrm{H}=$ $\left.0.4 \mathrm{~kJ} \mathrm{~mol}^{-1}\right)$ for insulin, which has been already reported 
for dendrimer-loaded insulin (Nowacka et al., 2016). The sequence of endothermic followed by exothermic peak could be correlated with protein denaturation followed by aggregation (Sarmento, Veiga, Ferreira, 2006). It is possible that insulin once denatured can expose interaction sites able to produce insulin-insulin linkages and, therefore, promote the formation of peptide aggregates (Gibson, Murphy, 2006).

The pattern of endothermic event followed by exothermic event was also observed for insulin loaded in the two compositions of chitosan/dextran sulfate nanoparticles studied. However, we can observe that the two types of nanoparticles show opposite behavior regarding the location and shape of the peaks. While chitosan/ dextran sulfate 1:9 nanoparticles had a shift of the endothermic peak to lower temperature $\left(\sim 67^{\circ} \mathrm{C}, \Delta \mathrm{H}=\right.$ $\left.0.7 \mathrm{~kJ} \mathrm{~mol}^{-1}\right)$ and a shift and broadening of the exothermic peak to higher temperature $\left(\sim 88^{\circ} \mathrm{C}, \Delta \mathrm{H}=1.0 \mathrm{~kJ} \mathrm{~mol}^{-1}\right)$, chitosan/ dextran sulfate $6: 4$ nanoparticles had a shift of both endothermic and exothermic peaks to higher temperatures, $88^{\circ} \mathrm{C}\left(\Delta \mathrm{H}=0.3 \mathrm{~kJ} \mathrm{~mol}^{-1}\right)$ and $94.5^{\circ} \mathrm{C}(\Delta \mathrm{H}$ $\left.=0.6 \mathrm{~kJ} \mathrm{~mol}^{-1}\right)$ respectively. These results suggested that insulin was, at least partially, protected from degradation in the cationic nanoparticle (chitosan: dextran sulfate, 6:4), which also presented the highest degree of insulin encapsulation.

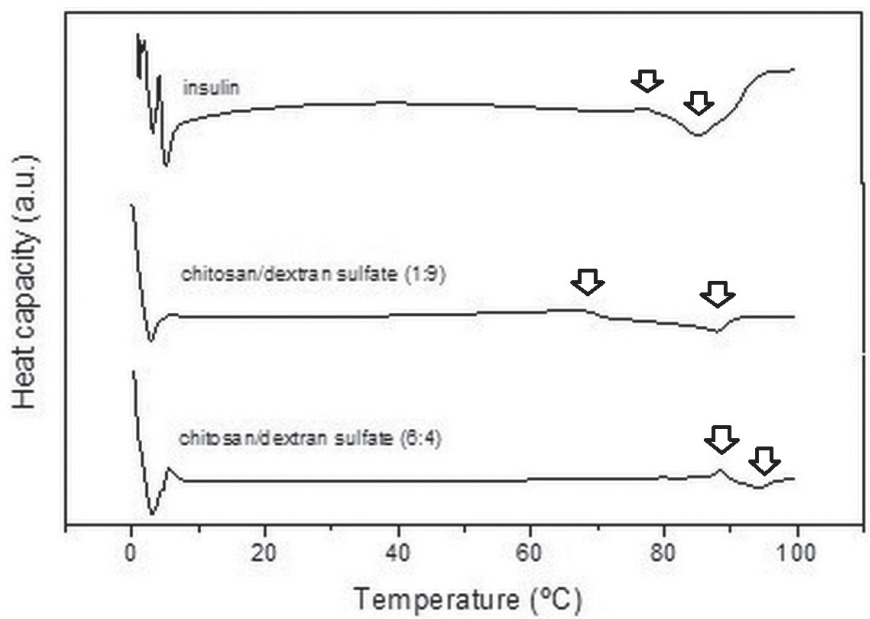

FIGURE 3 - Dynamic scanning calorimetry (DSC) curves of insulin (A), chitosan/dextran sulfate (1:9) nanoparticle (B) and chitosan/dextran sulfate $(6: 4)$ nanoparticle $(C)$, heated from 0 to $100{ }^{\circ} \mathrm{C}$.

In vitro drug release has been regarded as a parameter to assess formulation safety and efficacy, being used to reflect the in vivo behavior (Souza, 2014). Nanoparticles may serve as a platform for sustained release of drug, achieving a prolonged effect due to slow drug release, with several advantages, including lesser frequency of administration, reduced side effects, with consequent better patient compliance (Natarajan et al., 2014). Herein, the in vitro release studies using a dialysis membrane showed that both formulations presented similar release, with a slow pattern in the first $10 \mathrm{~h}$, then reaching the plateau (Figure 4). Sarmento, Veiga and Ferreira (2006) obtained similar results in their evaluation of chitosan/ dextran sulfate nanoparticles for oral delivery, however plateau was reached around $5 \mathrm{~h}$ in $\mathrm{pH} 6.8$ release medium. Therefore, the nanoparticles reported herein seem to present more sustained insulin release.

The process of drug release is complex and influenced by the physicochemical properties of the drug, the characteristics of the matrix and the release environment. Solute diffusion, polymeric material swelling and degradation have been suggested as the main factors explaining drug release. In general, mathematical models give insight into the release mechanism, revealing the process kinetics. Insulin release kinetics from the negatively charged nanoparticle corresponded to the Weibull model and is related to Fick's law diffusion, related to drug diffusion. On the other hand, the positively charged, chitosan/dextran sulfate (6:4) nanoparticle released insulin according to the Peppas model, an anomalous non-Fickian release kinetics, related to polymeric swelling (Grassi, Grassi, 2005). Hence, the chitosan/dextran sulfate ratio affected the kinetics mechanism of insulin release.

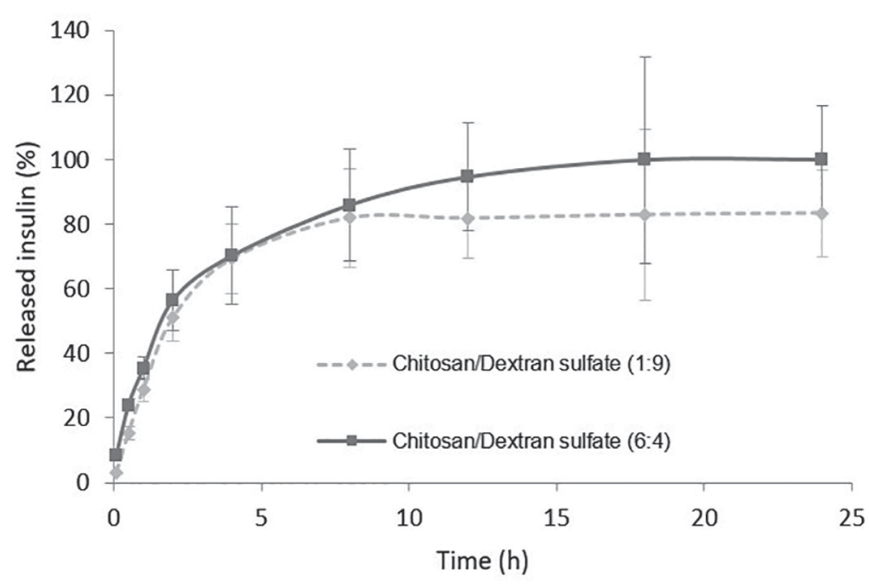

FIGURE 4 - In vitro release study of insulin-loaded nanoparticles.

Herein, it was shown the successful complexation with mucin, which was better for the chitosan/dextran sulfate (6:4) formulation, with maximum $66.72 \%$ interaction, compared to $34.70 \%$ interaction achieved with the chitosan/dextran sulfate (1:9) formulation, at 0.5 mucin/nanoparticle ratio (Figure 5). It is important to consider that the formulation prepared with more chitosan 
presents positive zeta potential, and it is known that positive surface has an important role on mucoadhesion, since the positive charge of nanoparticles can interact with the negatively charged sialic groups of mucin (Barbi et al., 2015). The high interaction with mucin presented by the positively charged nanoparticle might be an indicative of promising nasal or pulmonary applications.

Furthermore, it is interesting to note that the positively charged formulation interaction with mucin decreased in the presence of higher mucin/nanoparticle ratios, whereas the behavior is opposite for the negatively charged formulation, indicating that the electrostatic contribution for interaction decreases when the formulation is present in lower concentrations. On the highest mucin/ nanoparticle ratio, the percentage interaction with mucus is similar for the two formulations, around $40 \%$. It should be noted, on the other hand, that other parameters affect interaction between nanoparticles and mucus, for instance, buffer conditions such as $\mathrm{pH}$ and ionic strength (Lieleg, Vladescu, Ribbeck, 2010). Nanoparticles not only must be mucoadhesive, but also pass through mucus barrier to reach circulation. For mucus penetration, nanoparticles must be small enough to avoid steric inhibition by dense fiber mesh. In this context, it has been previously demonstrated that nanoparticles as large as $500 \mathrm{~nm}$ can rapidly diffuse though physiological human mucus (Lai et al., 2007). Therefore, the nanoparticles developed herein might have potential for mucus penetration.

The nasal or pulmonary routes represent promising alternative delivery sites, offering the possibility to avoid the first-pass metabolism enabling direct blood drug absorption. Noteworthy, the pulmonary route is particularly attractive for protein and peptide delivery owing to the large absorptive surface area with the thin alveolar mucosal membrane (Ahmed, Aljaeid, 2016). Albeit promising for the delivery of several drugs, these routes present the challenge of the mucociliary clearance (Alpar et al., 2005). Thus, mucoadhesive formulations emerged as an alternative to address this issue (Barbi et al., 2015). In this context, chitosan is one of the most employed biomaterials for the purpose of mucoadhesion, due to electrostatic interactions of cationic chitosan with negatively charged mucin (Sogias, Williams, Khutoryanskiy, 2008). Regarding insulin effect following in vivo administration, chitosan gels have caused increase in insulin absorption and reduction of glucose level after nasal administration (Varshosaz, Sadrai, Heidari, 2006). Another paper reported the preparation of a dry powder system consisting of microencapsulated insulin-loaded chitosan nanoparticles, which were evaluated in vivo in rats and resulted in deep lung deposition with pronounced and prolonged hypoglycemic effect (Ahmed, Aljaeid, 2016). On the other hand, chitosan-sulfate dextran is a novel approach for insulin delivery to the nasal or pulmonary routes and presents potential for future evaluation.

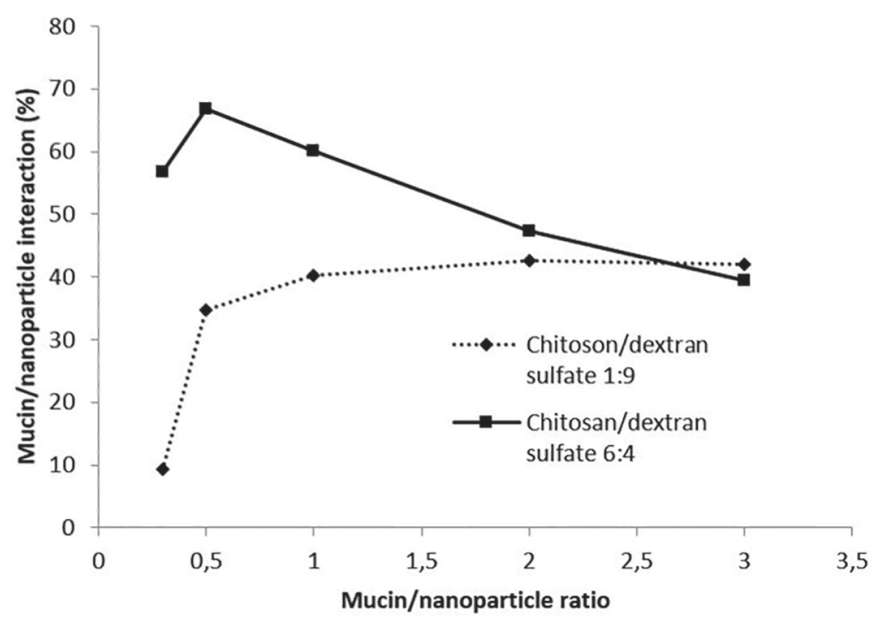

FIGURE 5 - Mucin and insulin-loaded nanoparticles interaction study.

Toxicology of nanoparticles is an important aspect to be evaluated before clinical application. Indeed, cytotoxicity studies have been widely employed as part of this evaluation (Yildirimer et al., 2011). In our studies, we evaluated the cytotoxicity potential of insulin and insulin-loaded nanoparticles against normal lung fibroblast cells. This cytotoxicity study showed that all nanoparticles had non-inhibitory effect on cells at the tested concentrations, with $\mathrm{IC}_{50}$ values greater than 250 $\mu \mathrm{g} \mathrm{mL} \mathrm{m}^{-1}$, as depicted in Figure 6, while doxorubicin, employed as positive control, presented IC50 at $4.5 \mu \mathrm{gmL}$ ${ }^{1}$. Hence, the relatively low cytotoxicity of the insulinloaded nanoparticles could be attributed to the presence of biocompatible polymers, which are responsible for weak interactions with the cell membrane (Lopes et al., 2016).

\section{CONCLUSION}

In this paper, we reported the development and characterization of nanoparticles based on chitosan/ dextran sulfate formed by polyelectrolytes condensation for insulin loading. Results demonstrated that the proper selection of polyelectrolyte total concentration, along with chitosan/dextran sulfate ratio, affected nanoparticles size and zeta potential. Although herein nanoparticles were able to encapsulate insulin with low efficiency, it was clear that positively charged nanoparticles based on chitosan/ dextran sulfate at the ratio of $6: 4$ better encapsulated the peptide compared to the composition at the ratio of 1:9, 
A)

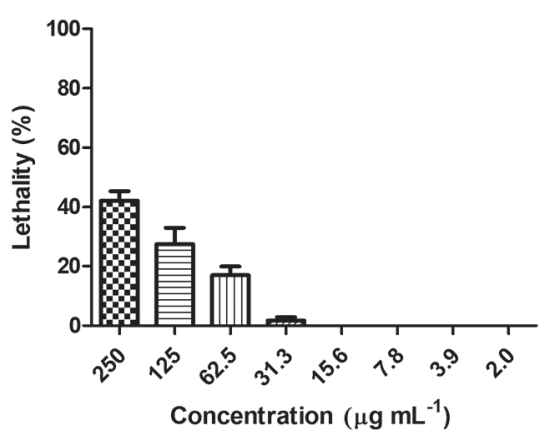

D)

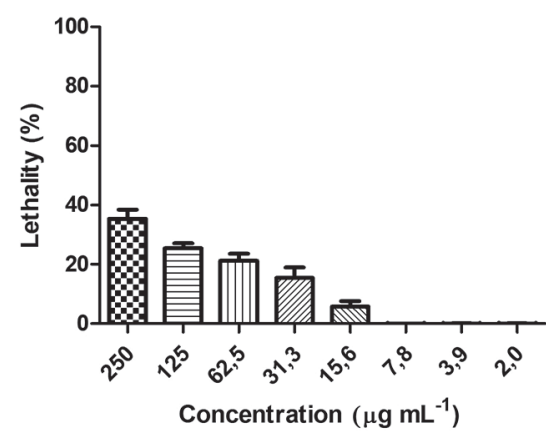

B)

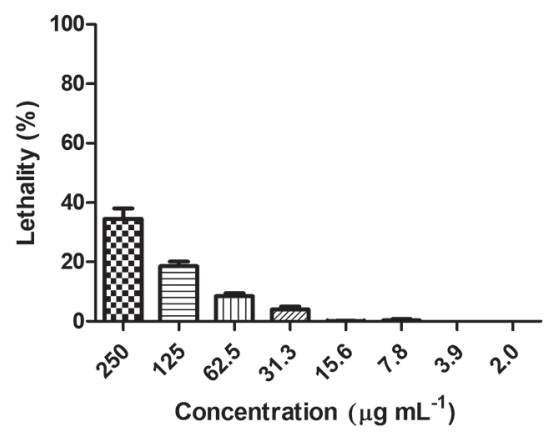

E)

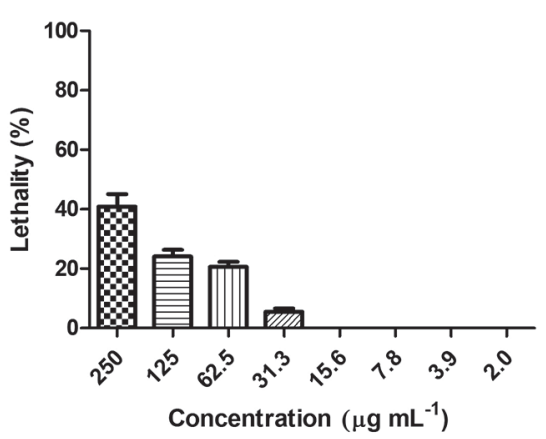

C)

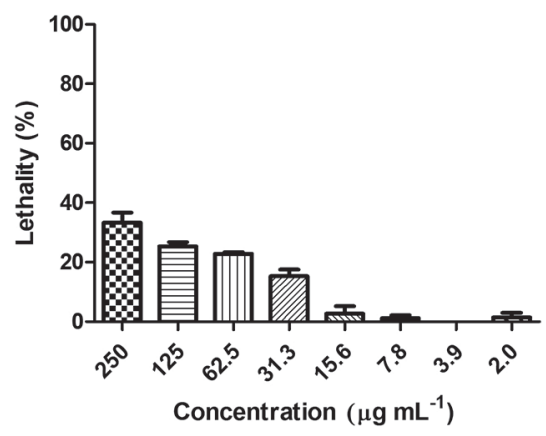

FIGURE 6 - Cytotoxicity evaluation on MRC-5 cells by the resazurin method in different concentrations of insulin (A), blank chitosan:dextran sulfate (1:9) nanoparticle (B), insulin-loaded chitosan:dextran sulfate (1:9) nanoparticle (C), blank chitosan:dextran sulfate (6:4) nanoparticle (D), insulin-loaded chitosan:dextran sulfate (6:4) nanoparticle (E).

due to excess chitosan. Insulin appeared to be partially protected from degradation when encapsulated, according to thermal analysis. Interestingly, insulin release from nanoparticles was sustained, particularly in the first $10 \mathrm{~h}$. It was demonstrated the efficient mucus complexation between mucin and nanoparticles, especially with the positivelycharged one, which could suggest potential application for nasal or pulmonary delivery. Finally, the insulin-loaded formulations exhibited no cytotoxicity potential against a lung cell line. Taken together, the results shown herein evidenced potential for future studies regarding insulinloaded chitosan/dextran sulfate nanoparticles for nasal or pulmonary delivery, potentially applied to treat diabetes.

\section{ACKNOWLEDGEMENTS}

This work was supported by Coordenação de Aperfeiçoamento de Pessoal de Nível Superior (CAPES), Fundação de Amparo à Pesquisa do Estado de São PauloFAPESP (Grant \#2014/24180-0), Conselho Nacional de Desenvolvimento Científico e Tecnológico (CNPq) and Programa de Apoio ao Desenvolvimento Científico da Faculdade de Ciências Farmacêuticas (PADC-FCFUNESP).

\section{REFERENCES}

Ahmed TA, Aljaeid BM. Preparation, characterization, and potential application of chitosan, chitosan derivatives, and chitosan metal nanoparticles in pharmaceutical drug delivery. Drug Des Devel Ther. 2016;10:483-507.

Alpar HO, Somavarapu S, Atuah KN, Bramwell VW. Biodegradable mucoadhesive particulates for nasal and pulmonary antigen and DNA delivery. Adv Drug Deliv Rev. 2005;57(3 Spec Issu):411-30.

Andreani T, Miziara L, Lorenzón EN, De Souza ALR, Kiill $\mathrm{CP}$, Fangueiro JF, et al. Effect of mucoadhesive polymers on the in vitro performance of insulin-loaded silica nanoparticles: Interactions with mucin and biomembrane models. Eur J Pharm Biopharm. 2015;93:118-26.

Barbi S, Carvalho FC, Kiill CP, Barud S, Santagneli SH, José $\mathrm{S}$, et al. Preparation and Characterization of Chitosan Nanoparticles for Zidovudine Nasal Delivery. J Nanosci Nanotechnol. 2015;15(1):865-74. 
Boya VN, Lovett R, Setua S, Gandhi V, Nagesh PKB, Khan S, Jaggi M, Yallupu MM, Chauhan, SC. Probin mucin interaction behavior of magnetic nanoparticles. J Coll Interf Sci. 2017;488:258-68.

Casettari L, Illum L. Chitosan in nasal delivery systems for therapeutic drugs. J Control Release. 2014;190:189-200.

Chaiyasan W, Srinivas SP, Tiyaboonchai W. Mucoadhesive chitosan-dextran sulfate nanoparticles for sustained drug delivery to the ocular surface. J Ocul Pharmacol Ther. 2013;29(2):200-207.

Delair T. European Journal of Pharmaceutics and Biopharmaceutics Colloidal polyelectrolyte complexes of chitosan and dextran sulfate towards versatile nanocarriers of bioactive molecules. Eur J Pharm Biopharm. 2011;78(1):10-8.

Diop M, Auberval N, Viciglio A, Langlois A, Bietiger W, Mura $\mathrm{C}$, et al. Design, characterisation, and bioefficiency of insulinchitosan nanoparticles after stabilisation by freeze-drying or cross-linking. Int J Pharm. 2015;491(1-2):402-8.

Fonte P, Araújo F, Silva C, Pereira C, Reis S, Santos HA, et al. Polymer-based nanoparticles for oral insulin delivery: Revisited approaches. Biotechnol Adv. 2014;33(6):1342-54.

Gibson TJ, Murphy RM. Inhibition of insulin fibrillogenesis with targeted peptides. Protein Sci. 2006;15(5):1133-41.

Giovino C, Ayensu I, Tetteh J, Boateng JS. Development and characterization of chitosan films impregnated with insulin loaded PEG-b-PLA nanoparticles (NPs): A potential approach for buccal delivery of macromolecules. Int J Pharm. 2012;428(12):143-51.

Grassi M, Grassi G. Mathematical modelling and controlled drug delivery : matrix systems. Curr Drug Deliv. 2005;2(1):97116.

Huang X, Du YZ, Yuan H, Hu FQ. Preparation and pharmacodynamics of low-molecular-weight chitosan nanoparticles containing insulin. Carbohydr Polym. 2009;76(3):368-73.

Iyer R, Hsia CCW, Nguyen, KT. Nano-therapeutics for the lung: state-of-the art and future perspectives. Curr Pharm Des. 2015;21(36):5233-5244.
Jain AK, Khar RK, Ahmed FJ, Diwan P V. Effective insulin delivery using starch nanoparticles as a potential trans-nasal mucoadhesive carrier. Eur J Pharm Biopharm. 2008;69(2):42635 .

Jorgensen L, Weert MVANDE, Vermehren C, Bjerregaard S, Frokjaer S. Probing structural changes of proteins incorporated into water-in-oil emulsions. J Pharm Sci. 2004;93(7):1847-59.

Lai SK, O’Hanlon DE, Harrold S, Man ST, Wang Y-Y, Cone RA, et al. Rapid transport of large polymeric nanoparticles in fresh undiluted human mucus. Proc Natl Acad Sci USA. 2007;104(5):1482-7.

Lieleg O, Vladescu I, Ribbeck K. Characterization of particle translocation through mucin hydrogels. Biophys J. 2010;98(9):1782-9.

Li L, Jiang G, Yu W, Liu D, Chen H, Liu Y, et al. Preparation of chitosan-based multifunctional nanocarriers overcoming multiple barriers for oral delivery of insulin. Mater Sci Eng C. 2017;70(Pt 1):278-86

Liu S, Jin M, Quan Y, Kamiyama F, Katsumi H. The development and characteristics of novel microneedle arrays fabricated from hyaluronic acid, and their application in the transdermal delivery of insulin. J Control Release. 2012;161(3):933-41.

Lopes M, Shrestha N, Correia A, Shahbazi MA, Sarmento B, Hirvonen J, et al. Dual chitosan/albumin-coated alginate/dextran sulfate nanoparticles for enhanced oral delivery of insulin. J Control Release. 2016;232:29-41.

Mao S, Bakowsky UDO, Jintapattanakit A, Kissel T. Selfassembled polyelectrolyte nanocomplexes between chitosan derivatives and insulin. J Pharm Sci. 2006;95(5):1035-48.

Natarajan JV, Nugraha C, Ng XW, Venkatraman S. Sustainedrelease from nanocarriers : a review. J Control Release 2014;193:122-38.

Nowacka O, Milowska K, Belica-pacha S, Palecz B. Generationdependent effect of PAMAM dendrimers on human insulin fibrillation and thermal stability. Int J Biol Macromol. 2016;82:54-60.

Ravindranathan S, Koppolu B, Smith SG, Zaharoff DA. Effect of chitosan properties on immunoreactivity. Mar Drugs. 2016;14(5):91-103. 
Sah SP, Singh B, Choudhary S, Kumar A. Animal models of insulin resistance: A review. Pharmacol Reports [Internet]. 2016;68(6):1165-77.

Salvioni L, Fiandra L, Del Curto MD, Mazzucchelli S, Allevi R, Truffi M, et al. Oral delivery of insulin via polyethylene iminebased nanoparticles for colonic release allows glycemic control in diabetic rats. Pharmacol Res. 2016;110:122-30.

Sarmento B, Veiga F, Ferreira D. Development and characterization of new insulin containing polysaccharide nanoparticles. Colloids Surf B: Biointerfaces. 2006;53(2):193202.

Schatz C, Domard A, Viton C, Pichot C. Versatile and efficient formation of colloids of biopolymer-based polyelectrolyte complexes. Biomacromulecules. 2004a;5(5):1882-92.

Schatz C, Lucas J, Viton C, Domard A, Pichot C, Delair T. Formation and properties of positively charged colloids based on polyelectrolyte complexes of biopolymers. Langmuir. 2004b;(6):7766-78.

Sintov AC, Levy HV, Botner S. Systemic delivery of insulin via the nasal route using a new microemulsion system: In vitro and in vivo studies. J Control Release. 2010;148(2):168-76.

Smith JM, Dornish M, Wood EJ. Involvement of protein kinase $\mathrm{C}$ in chitosan glutamate-mediated tight junction disruption. Biomaterials. 2005;26(16):3269-76.

Sogias IA, Williams AC, Khutoryanskiy V. Why is chitosan mucoadhesive? Biomacromolecules. 2008;9(7):1837-42.
Souza SD. A review of in vitro drug release test methods for nano-sized dosage forms. Adv Pharms. 2014;2014:304757.

Szekalska M, Czarnomysy R, Lavri` Z, Szyma E. Novel spray dried glycerol 2-phosphate cross-linked chitosan microparticulate vaginal delivery system - development, characterization and cytotoxicity studies. Mari Drugs. 2016;14(10):174-186

Varshosaz J, Sadrai H, Heidari A. Nasal delivery of insulin using bioadhesive chitosan gels. Drug Deliv. 2006;13(1):31-8.

Vrignaud S, Benoit JP, Saulnier P. Strategies for the nanoencapsulation of hydrophilic molecules in polymer-based nanoparticles. Biomaterials. 2011;32(33):8593-8604.

Whiting DR, Guariguata L, Weil C, Shaw J. IDF Diabetes Atlas: Global estimates of the prevalence of diabetes for 2011 and 2030. Diabetes Res Clin Pract. 2011;94(3):311-21.

Yildirimer L, Thanh NTK, Loizidou M, Seifalian AM. Toxicological considerations of clinically applicable nanoparticles. Nano Today. 2011;6:585-607.

Zhang X, Zhang H, Wu Z, Wang Z, Niu H, Li C. Nasal absorption enhancement of insulin using PEG-grafted chitosan nanoparticles. Eur J Pharm Biopharm. 2008;68(3):526-34.

Zheng C, Guo Q, Wu Z, Sun L, Zhang Z, Li C, et al. Amphiphilic glycopolymer nanoparticles as vehicles for nasal delivery of peptides and proteins. Eur J Pharm Sci. 2013;49(4):474-82.

Received for publication on $13^{\text {th }}$ June 2017 Accepted for publication on $18^{\text {th }}$ October 2017 